\title{
Factores sociodemográficos y económicos asociados a no realizar cuarentena por COVID-19 en población venezolana residente en Chile
}

\author{
Sociodemographic and economics factors associated with non-compliance of \\ the quarantine recommendation due to COVID-19 in Venezuelan migrants in \\ Chile
}

Pablo Ignacio Roessler-Vergara $\mathbb{D}^{1}$, Tomás Soto-Ramírez $\mathbb{D}^{1}$, Báltica Cabieses $\mathbb{D}^{2}$

${ }^{1}$ Fundación Servicio Jesuita a Migrantes, Chile.

2Universidad del Desarrollo, Instituto de Ciencias e Innovación en Medicina, Chile

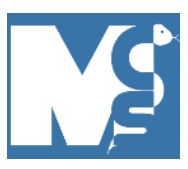

Recibido: $25 / 01 / 2021$

Revisado: $14 / 02 / 2021$

Aceptado:19/05/2021

\section{Autor correspondiente}

Pablo Ignacio Roessler Vergara Fundación Servicio Jesuita a Migrantes, Chile.

pablo.roessler@sjmchile.org

\section{Conflictos de interés}

Los autores declaran no poseer conflictos de interés.

\section{Fuente de financiación}

Los autores no recibieron apoyo financiero para la investigación, autoría y/o publicación de este artículo.

Este artículo es publicado bajo una licencia de Creative Commons

Reconocimiento 4.0 Internacional.

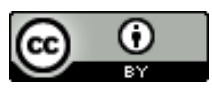

\section{RESUMEN}

Introducción: el colectivo venezolano residente en Chile ha aumentado en cantidad y en la vulnerabilidad social en que migran. Objetivo: analizar qué elementos sociodemográficos y económicos dificultan la realización de cuarentena en la población venezolana residente en Chile, considerando cómo esto varía según características como estar trabajando, tener seguro de salud, sexo y nivel educacional. Metodología: el diseño corresponde a un estudio cuantitativo observacional de corte transversal desde una encuesta online sobre COVID-19 a extranjeros en Chile, realizada en abril (2020), por medio de una estrategia de muestreo en "bola de nieve" ( $n=1,690$ migrantes). Este análisis secundario se focaliza en participantes venezolanos $(\mathrm{N}=1,006)$, por medio de análisis descriptivo, bivariado y de regresión multivariada, con ajuste de Raking para disminuir el sesgo de auto-selección. De estos, el 49,6\% fueron mujeres, y la mayoría (el 50\%) tenía entre 30 y 49 años. Resultados: la probabilidad de no poder realizar cuarentena es mayor en quienes cuentan con trabajo ([OR=5,35,95\%IC [3,16-9,02]), en relación a los que no; en quienes no poseen previsión de salud $([\mathrm{OR}=4,02,95 \% \mathrm{IC}[1,57-10,32])$ y en los que tienen previsión pública (Fonasa) ([OR=3,92, 95\%IC [1,84-8,35]), en relación a las personas con previsión privada; en hombres ([OR=2,23, 95\%IC [1,50-3,32]) que en mujeres; $y$ en los que tienen nivel educacional menor a nivel superior ([OR=1,74, 95\%IC [11,50-3,32]). Conclusión: la asociación encontrada entre no cumplir cuarentena con condiciones laborales y tipo de seguro de salud expone la relevancia de la vulnerabilidad socioeconómica en las oportunidades de llevar adelante medidas de cuidado de salud pública en población migrante venezolana en Chile, como es el seguimiento de confinamiento durante una pandemia. Esto es importante para la planificación sanitaria en futuras crisis socio-sanitarias.

Palabras clave: Migrantes; Condiciones de Trabajo; Beneficios en salud; Pacientes no Asegurados; pandemia; salud pública.

\section{ABSTRACT}

Introduction: The Venezuelan group residing in Chile has increased in number and in the social vulnerability in which they migrate. Objective: To analyze which sociodemographic and economic elements make it difficult to carry out quarantine in the Venezuelan population residing in Chile, considering how this varies according to characteristics such as being working, having health insurance, sex, and educational level. Methodology: Cross-sectional observational quantitative study from an online poll on COVID-19 to foreigners in Chile, carried out in April (2020), through a "snowball" sampling strategy ( $n=1,690$ migrants). This secondary analysis focuses on Venezuelan participants $(\mathrm{N}=1,006)$, through descriptive, bivariate and multivariate regression analysis, with Raking adjustment to reduce self-selection bias. Of these, $49.6 \%$ were women, and the majority (50\%) were between 30 and 49 years old. Results: The probability of not being able to quarantine is higher in those who have a job ([OR=5.35, 95\% $\mathrm{Cl}$ [3.16-9.02]), in relation to those who do not; in those who do not have a health insurance ([OR=4.02, 95\% $\mathrm{Cl}$ [1.57-10.32]) and in those who have public insurance (i.e. Fonasa) ([OR=3.92, 95\% Cl [ 1.84-8.35]), in relation to people with private pension; in men ([OR=2.23, 95\% Cl [1.50-3.32]) than in women; and in those with a lower educational level at a higher level ([OR=1.74, 95\% Cl [11.50-3.32]). Conclusion: The association found between not complying with quarantine due to working conditions and type of health insurance exposes the relevance of socioeconomic vulnerability in the opportunities to carry out public health care measures in the Venezuelan migrant population in Chile, such as monitoring of confinement during a pandemic. This is important for health planning in future socio-health crises.

Keywords: Migrants; Working Conditions; Health Benefits; Uninsured Patients; pandemic; public health. 


\section{INTRODUCCIÓN}

Para diciembre 2019, la población extranjera residente en Chile llegó a 1492 522, siendo el colectivo venezolano el más presente, representando el 30,5\% de los extranjeros que habitan el territorio (1). En los últimos años se ha dado un aumento del éxodo venezolano hacia diferentes países, incluido Chile, pero también se ha incrementado la vulnerabilidad social de dicho flujo migratorio $(2,3)$, por ello se ha vuelto una población que es de interés estudiar. Considerando esto último, el presente artículo busca aproximarse a la manera en que migrantes venezolanos en Chile perciben que han enfrentado la pandemia de COVID19. Más específicamente, si han podido cumplir la cuarentena, que ha sido una de las medidas más promulgadas a nivel mundial para proteger a la población del contagio y los peligros en materia de salud pública que ello conlleva. De esta manera, el propósito de estudio fue analizar barreras sociodemográficas y económicas que han enfrentado migrantes venezolanos en Chile para cumplir con esta medida de salud pública.

Respecto a la evidencia disponible sobre esta temática a la fecha, según incipientes investigaciones internacionales las medidas de confinamiento han sido más complejas de cumplir en poblaciones socialmente vulnerables. Por un lado, exponen la dificultad de llevarla a cabo en hogares hacinados con bajo distanciamiento social, donde diferentes núcleos familiares comparten el mismo espacio doméstico (46). También se describe que la inseguridad e informalidad laboral, así como el nivel de ingresos familiares al momento de cumplir con la indicación de confinamiento, son variables relevantes en la percepción de enfrentar la pandemia y en la experiencia de cumplir con las recomendaciones (5-7).

En reconocimiento de este cuerpo de conocimiento aun en desarrollo, este estudio se interesa en investigar algunas barreras en el acceso a derechos tales como salud y medidas de cuidado en la población migrante, incluyendo dimensiones cruciales como oportunidad de información y tenencia de previsión/seguro de salud $(8,9)$. Cabe destacar que la dimensión de tipo de previsión de salud en Chile es relevante, dado que el sistema de salud chileno es segmentado y fragmentado entre público y privado, y la oportunidad de acceso a uno u otro está determinado por en nivel socioeconómico de la persona (10).

Por otro lado, también se ha descrito en la literatura que, en la población extranjera residente en Chile, ser mujer se asocia en mayor medida con el uso de consultas médicas. Esto pudiera reflejar necesidades de salud específicas que ellas enfrentan como salud materna, violencia, y salud sexual y reproductiva. También podría representar mayor necesidad de atención en salud y una cultura de relación con el sistema de salud que podría facilitar el uso de servicios de salud (8).

El nivel educacional en población migrante, por su parte, no siempre representa un factor protector, pues que migrantes altamente capacitados no siempre consiguen condiciones de vida equivalentes a sus calificaciones $(11,12)$. Por esta razón, fue de interés de este estudio el analizar posibles asociaciones entre nivel educacional y capacidad de realizar cuarentena.

Con lo anterior, el objetivo del presente estudio fue analizar la relación entre un conjunto de variables sociodemográficas y económicas con la realización de cuarentena en población venezolana residente en Chile, esperando contribuir al conocimiento actual en materia de migración y pandemia en la región.

\section{METODOLOGÍA}

El presente artículo emanó de un estudio cuantitativo observacional de corte transversal que utiliza como fuente la Encuesta sobre COVID-19 a poblaciones migrantes internacionales en Chile, realizada las primeras tres semanas del mes de abril 2020 (cuando comenzaron a regir medidas obligatorias de cuarentena en dicho país). La encuesta fue ejecutada (y luego facilitada a los autores de este artículo) por el Programa de Estudios Sociales en Salud (PROESSA) ICIM de la Universidad del Desarrollo, junto a organizaciones de la sociedad civil (13).

Los criterios de inclusión en la encuesta fueron: ser migrante internacional residiendo en Chile mayor de 18 años y tener la voluntad libre e informada de participar. Se realizó por medio de la plataforma Google Form, estando disponible por 21 días en un link seguro que, una vez cumplido el período, desapareció.

Presentó un muestreo no probabilístico con técnica "bola de nieve", cuya "semilla" o inicio fue la difusión desde organizaciones migrantes/pro migrantes y de la red asistencial de salud pública de Chile a nivel nacional. Así el link de la encuesta se compartió con un afiche informativo de la encuesta por medio de redes sociales, en particular en grupos de WhatsApp, Twitter y Facebook de la Facultad de Medicina Clínica Alemana UDD, Servicio Jesuita a Migrantes (SJM), INCAMI, Núcleo Mileno MICROB-R, Chile Científico y organizaciones de migrantes y pro migrantes. Luego se traspasó entre redes cercanas de cada encuestado, persona a persona, por medio de WhatsApp entre 
grupos de migrantes de diversos colectivos migrantes en el país. De esta forma, no hubo contacto directo con información personal de potenciales participantes, sino que solo a través de redes sociales a las cuales los mismos participantes adherían al momento de realizarse el estudio. Se escogió esta técnica dada las dificultades para llegar a la población migrante en un contexto de pandemia, convirtiéndola en una población difícil de contactar $(14,15)$. El tamaño muestral alcanzó los 1,690 migrantes (42 de Argentina; 26 de Bolivia; 27 de Brasil; 229 de Colombia; 48 de Cuba; 27 de Ecuador; 12 de España; 91 de Haití; 10 de México; 74 de Perú; 25 de República Dominicana; 1,006 de Venezuela; 69 de Otros Países y 4 sujetos que no indicaron nacionalidad).

Acorde al objetivo de estudio, el análisis se centró en los 1,006 venezolanos participantes por las siguientes razones que los han vuelto un colectivo de interés para estudiar: 1) es la población que más ha aumentado su flujo migratorio a Chile en los últimos años $(1,3) ; 2$ ) las condiciones de migración han sido cada vez más precarias convirtiéndose en una población que requiere de protección internacional según señalan diferentes organismos internacionales $(2,3)$; 3) la última estimación poblacional de personas extranjeras que habitan Chile (a 2019), les expone como el colectivo migrante mayoritario, representando el $30.5 \%$ del total de población extranjera en Chile. De ellos, el $50,4 \%$ son hombres y $49,6 \%$ son mujeres (1).

Se presenta como variable dependiente la no realización de cuarentena: una variable binaria cuyas categorías de respuesta fueron $1=$ No y $0=$ Sí. Se enumeró con 1 la opción "No" para conocer de manera más sustantiva las barreras para realizar confinamiento. Las variables independientes y sus alternativas de respuesta fueron: 1 ) Sexo: $0=$ Mujer $y$ 1=Hombre; 2) Situación ocupacional: Sí estoy trabajando; No, pero no quiero trabajar; y No, pero quiero trabajar. Se recodificó en dos categorías, según si se encuentra trabajando: $0=$ No; $1=S i ́$; 3) Tipo de previsión o seguro de salud: No sé; No tengo ninguno; Sí, Fonasa (Fondo Nacional de Salud, modelo público); Sí, Isapre (Instituciones de Salud Previsional, modelo privado); Sí, otro (seguro internacional u otro). Se reagruparon en 3 categorías: $0=$ Privado (Isapre 0 seguro internacional); $1=$ Público (Fonasa); $2=$ No tiene o no sabe; 4) Nivel educacional: Primario, Secundario y Universitario. Se agruparon en $0=$ Universitario; 1=Secundaria o menos; 5) Manejo de información "Ante cualquier inquietud sobre COVID-19, ¿sabe dónde consultar?" (0=Sí; 1=No); 6) Tramos etarios. Se consideró edad como variable clave para el control estadístico, llevándose a tramos: $0=50$ años o más;
$1=30$ a 49 años; $2=18$ a 29 años. 7) Tiempo en Chile. $0=$ Menos de un año; $1=$ Más de un año. La decisión para agrupar, tanto la variable de tramos etarios como de tiempo en Chile, responde a la distribución de ambas variables, como también a la manera en la que se encontraban las alternativas en el cuestionario (estaban por categorías y no de forma numérica). La variable edad al ingresar al modelo de regresión permitió, cómo se verá más adelante, una mejor interpretación.

Luego del levantamiento, se realizó un ajuste de Raking para disminuir el riesgo de sesgo de selección, utilizando como variables poblacionales el sexo y la edad de los venezolanos residentes en Chile a diciembre 2019 (1). El proceso consistió en ajustar iterativamente el peso para cada participante de estudio, hasta que la distribución de la muestra se alineara con la población general venezolana estimada en Chile para dichas variables. Si bien el Raking no soluciona totalmente el sesgo de autoselección, permite observar una distribución más cercana a estimaciones nacionales en términos de edad $(34,6 \%$ para 18 a 29 años; $43,9 \%$ para 30 a 49 años y $9,1 \%$ para el segmento de 50 años y más) y sexo (50,4 \% para hombres y 49,6\% para mujeres) de la población venezolana que habita Chile. Además, se ha demostrado la utilidad de este ajuste en muestras no probabilísticas como la de este estudio (16). Las características de la muestra se conocerán en la primera parte del apartado de resultados.

Una vez realizado el ajuste, se realizó análisis univariado y bivariado. En este segundo se utilizó la prueba de asociación chi-cuadrado entre las variables independientes y dependientes como primera aproximación. Luego se llevó un análisis multivariado, con modelos de regresión logística, utilizando dos estimadores: Odds Ratio (OR) y chances. Los OR expresan la razón de probabilidades que un evento determinado ocurra en relación a que se presente uno distinto, y se utilizó dada su prevalencia en los estudios con variables categóricas $(17,18)$. Las chances o probabilidades, por su parte, se calculan como [(OR$\left.1)^{*} 100\right]$, con el fin de dar una interpretación más sustantiva al hecho de no realización de cuarentena (17). Se utilizó el software R para el ajuste de la encuesta, y Stata 16 para los análisis posteriores.

Relevante es señalar que la encuesta contó con la aprobación de un comité ético-social conformado por migrantes internacionales, como también con expertos en trabajo comunitario y derechos humanos. Siguiendo lineamientos éticos internacionales, la encuesta online presentó un consentimiento informado, donde se señalaba la libertad y autonomía de participar, que el 
manejo de información es anónimo y se describieron los productos científicos a alcanzar.

\section{RESULTADOS}

Un 23,4\% de las personas venezolanas encuestadas no había realizado cuarentena al momento de la aplicación. Por su parte el $56,1 \%$ se encontraba trabajando, y en cuanto a la previsión de salud, un 24,7 \% señaló no tener o no saber que cuentan con ella, un $62 \%$ Fonasa/Público, y el $13,1 \%$ Isapre/Privado. Un poco más de un tercio $(34,9 \%)$ señaló no saber a quién consultar en caso de dudas relacionadas al COVID-19, y el 31,4 \% llevaba menos de un año viviendo en Chile. El $49,6 \%$ fueron mujeres y un $75,1 \%$ contaba con nivel educacional superior. Finalmente, un $40 \%$ tenía entre 18 a 29 años, $50 \% 30$ a 49, y un $10 \% 50$ o más.

Ahora bien, la Tabla 1 expone las características del grupo que pudo realizar cuarentena, como también de quienes no pudieron. Así se puede saber que, de quienes realizaron cuarentena el 55, $1 \%$ eran mujeres, mientras que en quienes no realizaron, solo un tercio
$(33,2 \%)$ fueron mujeres. Por su parte, de quienes realizaron cuarentena el $48,6 \%$ se encontraba trabajando, mientras que este porcentaje fue de $80 \%$ en quienes no llevaron a cabo medida de confinamiento. En quienes realizaron cuarentena hay mayor porcentaje de quienes tienen seguro de salud privada y menor de quienes tienen seguro público. En cuanto a nivel educacional, quienes sí realizaron medida de confinamiento tienen un mayor porcentaje de personas con nivel educacional universitario o superior $(77,8 \%)$ que quienes no realizaron cuarentena (66,1\%).

También se observa que, de quienes realizaron cuarentena, el 67,2 \% señaló si saber dónde acudir ante inquietudes asociadas al COVID-10 en el marco de la pandemia, lo que fue menor en quienes no llevaron a cabo la medida (58,2 \%). Finalmente, los que sí realizaron confinamiento se ubican en mayor proporción en el tramo joven de 18 a 29 años (42 \% en comparación con $39,4 \%$ ), y en menor medida en el tramo de 50 años o más.

TABLA 1. CARACTERÍSTICAS RELEVANTES DE LA POBLACIÓN MIGRANTE Y SU ASOCIACIÓN CON EL HECHO DE NO REALIZAR CUARENTENA ${ }^{\mathrm{a}}$

\begin{tabular}{|c|c|c|c|c|c|c|}
\hline & \multicolumn{6}{|c|}{ Realización de Cuarentena } \\
\hline & & Sí & & No & & Valor de $\mathbf{p}^{b}$ \\
\hline \multirow{4}{*}{ Sexo } & & $\mathrm{n}$ & $\%$ & $\mathrm{n}$ & $\%$ & \multirow{4}{*}{$<0,001$} \\
\hline & Mujer & 425 & 55,1 & 78 & 33,2 & \\
\hline & Hombre & 346 & 44,9 & 157 & 66,7 & \\
\hline & Total & 770 & 100,0 & 236 & 100,0 & \\
\hline \multirow[t]{3}{*}{ Se encuentra trabajando } & No & 396 & 51,4 & 46 & 19,7 & \multirow[t]{2}{*}{$<0,001$} \\
\hline & Sí & 374 & 48,6 & 189 & 80,3 & \\
\hline & Total & 770 & 100,0 & 236 & 100,0 & \multirow{3}{*}{$<0,001$} \\
\hline \multirow[t]{2}{*}{ Tiene seguro de salud } & No tiene o no sabe & 198 & 25,7 & 51 & 21,5 & \\
\hline & Público (FONASA) & 456 & 59,2 & 170 & 71,8 & \\
\hline \multirow{5}{*}{ Nivel educacional } & Privado & 117 & 15,1 & 16 & 6,6 & \multirow{5}{*}{$<0,001$} \\
\hline & Total & 770 & 100,0 & 236 & 100,0 & \\
\hline & Secundaria o menor & 171 & 22,2 & 80 & 33,8 & \\
\hline & Educación Universitaria & 599 & 77,8 & 156 & 66,1 & \\
\hline & Total & 770 & 100,0 & 236 & 100,0 & \\
\hline \multirow[t]{2}{*}{ Manejo de la información } & Sí & 518 & 67,2 & 137 & 58,2 & \multirow[t]{2}{*}{0,009} \\
\hline & No & 252 & 32,8 & 99 & 41,8 & \\
\hline \multirow{4}{*}{ Tiempo en Chile } & Total & 770 & 100,0 & 236 & 100,0 & \multirow{4}{*}{0,344} \\
\hline & Menos de 1 año & 248 & 32,2 & 68 & 29,0 & \\
\hline & Más de un año & 522 & 67,8 & 167 & 71,0 & \\
\hline & Total & 770 & 100,0 & 236 & 100,0 & \\
\hline Tramos etarios & 18 a 29 años & 303 & 39,4 & 99 & 42,0 & 0.349 \\
\hline
\end{tabular}




$\begin{array}{lcccc}30 \text { a } 49 \text { años } & 384 & 49,9 & 119 & 50,4 \\ 50 \text { años o más } & 83 & 10,7 & 18 & 7,6 \\ \text { Total } & 770 & 100,0 & 236 & 100,0\end{array}$

Fuente: elaboración propia a partir de la Encuesta sobre Covid-19 a Poblaciones Migrantes Internacionales en Chile desarrollada por el Programa de Estudios Sociales en Salud.

a El total del cruce de las variables independientes con la dependiente no siempre da el n de la muestra, por lo tanto, se sugiere utilizar los porcentajes.

b Prueba Chi Cuadrado

La Tabla 2 da cuenta del modelo de regresión logística de asociación entre no haber cumplido la cuarentena y un conjunto de variables. El método utilizado fue el de máxima verosimilitud, el pseudo $\mathrm{R}^{2}$ utilizado fue el Nagelkerke porque equivale al ajuste de Cox y Snell y permite una mejor interpretación porque sus valores van de 0 a 1 . En este sentido, el pseudo $R^{2}$ de Nagelkerke del modelo crudo fue de 0,213 y el ajustado ( $\sin$ la variable edad) fue de 0,215 . Finalmente, el porcentaje de clasificación del primer modelo fue de $78,5 \%$ y del segundo un $76,6 \%$. Para comparar modelos de regresión con variable dependiente categórica es importante conocer el BIC (Bayesian Information Criteria), el cual mientras más pequeño expone mejor ajuste (18). Mientras que el BIC del modelo crudo es de 1,005, el del modelo ajustado fue de 991. Se eligió, en este sentido, el modelo que no posee la variable edad dado su mejor ajuste y medidas de significación estadística.

Así, en el modelo ajustado se observa que la no realización de cuarentena tuvo como asociación más importante la tenencia de empleo ([OR=5,32; 95\%IC $[3,16-9,02])$, donde quienes se encuentran trabajando presentan $435 \%$ más probabilidades de no realizar cuarentena que quienes no trabajan. Por otra parte, comparado con quienes tienen previsión privada de salud (Isapre), no contar con previsión de salud aumentó en $302 \%$ las chances de no realizar cuarentena ([OR=4,02; $95 \% \mathrm{IC}[1,57-10,32])$, lo mismo con quienes tienen Fonasa que presentan $292 \%$ más probabilidades en comparación a quienes tienen un seguro de salud privado ([OR=3,92, $95 \% \mathrm{IC}[1,84-8,35])$. Se observa también que los hombres tienen $123 \%$ más posibilidades que las mujeres de no realizar cuarentena ([OR=2,23; $95 \%$ IC [1,50-3,32]).

Lo propio ocurre con nivel educativo, donde quienes cuentan con educación secundaria o menor presentan $74 \%$ más probabilidades de no realizar cuarentena que quienes tienen educación superior ([OR=1,74; $95 \% \mathrm{IC}$ [11,50-3,32]). Finalmente, los que no sabían a quién acudir ante cualquier duda en el marco de la pandemia presentaron $29 \%$ más de posibilidades de no realizar cuarentena que quienes sí ([OR=1,29, $95 \%$ IC [0,84$1,96])$, no existiendo para este caso una asociación estadísticamente significativa.

TABLA 2: MODELOS DE REGRESIÓN LOGÍSTICA PARA EXPLICAR EL HECHO DE NO REALIZAR CUARENTENA PARA LA POBLACIÓN VENEZOLANA EN CHILE.

\begin{tabular}{|c|c|c|c|c|c|c|}
\hline Variable & OR crudo & Valor $\mathrm{p}$ & IC $95 \%$ & OR Ajustado & Valor de $\mathrm{p}$ & IC $95 \%$ \\
\hline \multicolumn{7}{|l|}{ Se encuentra trabajando } \\
\hline Sí & 5,32 & $<0,001$ & $3,15-8,99$ & 5,35 & $<0,001$ & $3,16-9,04$ \\
\hline No & Ref & Ref & Ref & Ref & Ref & Ref \\
\hline \multicolumn{7}{|l|}{ Tiene seguro de salud } \\
\hline Privado & Ref & Ref & Ref & Ref & Ref & Ref \\
\hline Público & 3,96 & $<0,001$ & $1,86-8,44$ & 3,92 & $<0,001$ & $1,84-8,35$ \\
\hline No tiene o no sabe & 4,09 & 0,004 & $1,58-10,54$ & 4,02 & 0,004 & $1,57-10,32$ \\
\hline \multicolumn{7}{|l|}{ Sexo } \\
\hline Hombre & 2,23 & $<0,001$ & $1,49-3,31$ & 2,23 & $<0,001$ & $1,50-3,32$ \\
\hline Mujer & Ref & Ref & Ref & Ref & Ref & Ref \\
\hline \multicolumn{7}{|l|}{ Nivel Educacional } \\
\hline Secundaria o menor & 1,73 & 0,048 & $1,00-2,98$ & 1,74 & 0,036 & $1,04-2,93$ \\
\hline $\begin{array}{l}\text { Educación } \\
\text { Universitaria }\end{array}$ & Ref & Ref & Ref & Ref & Ref & Ref \\
\hline
\end{tabular}


Factores sociodemográficos y económicos asociados a no realizar cuarentena por COVID-19

\begin{tabular}{ccccccc} 
Sí & Ref & Ref & Ref & Ref & Ref & Ref \\
No & 1,28 & 0,258 & $0,84-1,96$ & 1,29 & 0,244 & $0,84-1,96$ \\
Edad & & & & & - \\
18 a 29 años & Ref & Ref & Ref & - & - & - \\
30 a 49 años & 1,00 & 0,978 & $0,72-1,41$ & - & - \\
50 o más & 0,85 & 0,613 & $0,46-1,57$ & - & - \\
\hline \hline
\end{tabular}

OR: Odds Ratio

IC95\%: Intervalo de confianza al $95 \%$

Ref.: Categoría de referencia

\section{DISCUSIÓN}

Los resultados de este análisis sugieren aproximaciones para entender dificultades que han tenido las y los migrantes venezolanos residentes en Chile para la realización de cuarentena. En cuanto a las variables más relevantes se encontraron dos fundamentales, la tenencia de empleo y el tipo de previsión.

Respecto de la tenencia de empleo, destaca su importancia general para la probabilidad de no cumplir con la cuarentena recomendada, lo que puede dar cuenta de dificultades para adaptar las funciones laborales en contextos de crisis socio-sanitarias como una pandemia (19). De hecho, se ha observado cómo en Chile el servicio de trabajo informal de delivery de comidas y elementos básicos ha sido dominado por población migrante, incluyendo la venezolana. En dicha línea se debe prestarse atención a la informalidad y a riesgos ocupacionales asociados al trabajo en tiempo de pandemia $(20,21)$.

La segunda variable relevante de este estudio fue tipo de previsión de salud. A nivel mundial los migrantes internacionales tienden a presentar menores niveles de acceso a sistemas de salud y uso efectivo de prestaciones en países receptores $(22,23)$. Evidencia nacional documenta la centralidad de contar con previsión formal de salud para el uso efectivo de servicios de salud y medidas de cuidado $(8,9)$. El tema de acceso formal y uso efectivo del sistema de salud por parte de migrantes en Chile se renueva a la luz de estos resultados, donde los riesgos de no cumplir confinamiento también pueden asociarse a contar o no con inscripción al sistema de salud y el tipo de previsión que se tenga. Esto es relevante para la elaboración de estrategias a poblaciones migrantes en Chile y la región, velando por el principio de justicia de proteger a todos y todas en salud y a "no dejar a nadie atrás".

Por otra parte, pese a no haber sido significativa la asociación entre contar con información sobre COVID19 y el no haber realizado cuarentena, es relevante señalar que el manejo acerca de diversos aspectos de la sociedad receptora se ha documentado como relevante para la experiencia de inclusión social $(8,24)$. Esto reflejaría la importancia de ahondar en la investigación de esta dimensión en la vida de migrantes internacionales en Chile en general, y de población extranjera de origen venezolano en particular. Este estudio es pionero en su tipo en Chile y permite aproximarse a conocer cómo ciertas vulnerabilidades socioeconómicas, en este caso en migrantes venezolanos, dificultan el cumplimiento de medidas de cuidado, en el contexto de pandemia por el SARS-CoV2.

Pese a ello, es importante señalar las limitaciones que presenta, tanto en términos de representatividad como en las preguntas utilizadas. En cuanto a lo primero, si bien, gracias a la cooperación de diferentes organizaciones, la encuesta fue respondida por más de 1000 migrantes residentes en todas las regiones de Chile, al ser una muestra no probabilística (y a pesar de los ajustes estadísticos realizados con posterioridad para corregir), no se permite hacer inferencia directa de los resultados a la población venezolana residente en Chile. Sin embargo, como primer estudio de su tipo en Chile permite una primera aproximación al fenómeno que se busca estudiar.

Por su parte, las limitaciones asociadas a las preguntas se relacionan con el carácter breve de la encuesta (cuyo objetivo era ser eficiente, evitar preguntas sensibles que dificultaran la respuesta y llegar a un mayor tamaño de muestra). Por ejemplo, solo se podía conocer si la persona se encontraba trabajando o no, pero no el tipo de empleo, condiciones, o formalidad de éste. Estas limitaciones son importantes para futuros estudios, de manera que puedan integrar preguntas más detalladas que permitan mayor profundidad de análisis. Por ejemplo, incluir estatus de refugiado/asilado, experiencia migratoria, ingreso por paso habilitado/no habilitado, razones para migrar, nuevas preguntas laborales, redes de apoyo, entre otras.

De igual modo, los resultados de este análisis son útiles para el contexto migratorio y de crisis sanitaria, entregando insumos para acciones que tengan por 
objetivo proteger la salud de migrantes venezolanos en Chile, que, como se señaló, se han tornado en población de interés producto del aumento de su migración a dicho país, como también por la vulnerabilidad en la que migran y arriban. En particular es un material de apoyo para medidas que apunten a que esta población pueda cumplir indicaciones de la autoridad sanitaria sobre cuarentena cuando sea requerido, por medio de robustecer la protección social y laboral de estas personas.

Incluir migrantes en medidas nacionales de apoyo como canastas de alimentos y bonos familiares debe darse independiente de su estatus regulatorio. Solo así podremos promover que estas personas puedan cumplir con las indicaciones sanitarias en contextos de pandemia, lo cual tiene efectos positivos en el total de la población. Los resultados de este estudio son relevantes para Chile y la región, al informar sobre cómo la condición de trabajo, el tipo de previsión de salud y el nivel educacional pueden asociarse al hecho de no cumplir la cuarentena recomendada en contextos de pandemia por parte de migrantes venezolanos, que habitan todo el territorio latinoamericano en este momento. El éxodo humanitario venezolano es una crisis humanitaria de proporciones que ha impactado toda la región y que requiere de mayor comprensión y capacidad de respuesta en todos los países, en especial en escenarios de crisis sociosanitarias. Este estudio informa de variables migratorias y socioeconómicas modificables que están afectando y vulnerando la experiencia de protección y prevención de COVID-19 en migrantes venezolanos en Chile y que pueden ser compartidas con migrantes de otros países de la región. Así, estos hallazgos son útiles para aproximarse a cómo otros grupos poblacionales con características similares han enfrentado la medida de confinamiento para prevenir contagios.

Dadas las características que está teniendo la migración venezolana hacia Chile (2), tanto comunidades de ese mismo país que han emigrado a otras latitudes, como personas que salen de sus países debido a crisis humanitarias (solicitantes de asilo y refugio), pueden vivir situaciones similares al momento de llevar medidas de prevención ante contagios de la COVID-19 como es la realización de cuarentena (7).

Al mismo tiempo, se debe continuar avanzando con lo que la política de salud de migrantes se propuso en Chile, referida a acortar brechas de acceso formal a la salud pública y medidas de cuidado, independiente de país de origen, tiempo de residencia o estatus migratorio. No contar con acceso formal al sistema de salud sigue estando asociado a menores capacidades de inclusión social, como el no poder cumplir con medidas de confinamiento cuando se requiera en Chile. La salud de todos y todas depende de nuestra capacidad de sopesar estas brechas injustas, prevenibles y modificables.

Futuras investigaciones podrían ahondar en estos resultados apoyando a comprender con mayor profundidad cómo migrantes internacionales de origen venezolano están viviendo en Chile y cómo ayudarlos a cuidar mejor de sí y de los suyos en contextos de crisis sociales y sanitarias, con el fin de no dejar a nadie atrás.

\section{CONTRIBUCIÓN DE LOS AUTORES}

PRV: Concepción y diseño del estudio, procesamiento y análisis de datos, redacción artículo, revisión de la literatura, revisión crítica del artículo y aprobación versión final. TSR: Procesamiento y análisis de datos, redacción del artículo, revisión crítica del artículo y aprobación versión final. BC: Concepción y diseño del estudio, redacción artículo, revisión de la literatura, revisión crítica del artículo y aprobación versión final

\section{REFERENCIAS}

1. Instituto Nacional de Estadísticas, Departamento de Extranjería y Migración. Estimación de personas extranjeras residentes habituales en Chile al 31 de Diciembre de 2019. 2020.

2. Servicio Jesuita a Migrantes. Dinámicas fronterizas en el norte de Chile el año 2020: Pandemia, medidas administrativas y vulnerabilidad migratoria. 2020.

3. Servicio Jesuita a Migrantes. Migración en Chile. Anuario 2020. Medidas migratorias, vulnerabilidad y oportunidades en un año de pandemia Área. 2021.

4. Cao Y, Liu R, Qi W, Wen J. Spatial heterogeneity of housing space consumption in urban China: Locals vs. inter-and intra-provincial migrants. Sustainability .2020;12 (12):1-26. https://dx.doi.org/10.3390/su12125206

5. Koh D. Migrant workers and COVID-19. Occup Environ Med .2020;77:634-6. https://dx.doi.org/10.1136/oemed-2020106626

6. Ramírez-Cervantes KL, Romero-Pardo V, Pérez-Tovar C, Martínez-Alés G, Quintana-Diaz M. A medicalized hotel as a public health resource for the containment of Covid-19: more than a place for quarantining. J Public Health .2020:114. https://dx.doi.org/10.1093/pubmed/fdaa129

7. Poole DN, Escudero DJ, Gostin LO, Leblang D, Talbot EA. Responding to the COVID-19 pandemic in complex humanitarian crises. Int J Equity Health .2020;19 (1):1-3. https://dx.doi.org/10.1186/s12939-020-01162-y

8. Cabieses B. Salud y migración: un proceso complejo y multidimensional. In: Rojas Pedemonte $\mathrm{N}$, Vicuña JT, editors. Migr. En Chile Evid. Mitos Una Nueva Real., Santiago de Chile: LOM ediciones; 2019, p. 400.

9. Benítez A, Velasco C. Desigualdades en salud: Brechas en acceso y uso entre locales e inmigrantes. In: Aninat I, Vergara R, editors. Inmigr. En Chile Una Mirada Multidimens., Santiago de Chile: Fondo de Cultura Económica; 2019, p. 191-235.

10. Cotlear D, Gómez-Dantés O, Knaul F, Atun R, Barreto ICHC, Cetrángolo O, et al. La lucha contra la segregación social en la atención de salud en América Latina. MEDICC 
Rev .2015;17:S40-52. https://dx.doi.org/10.1016/S01406736(14)61647-0

11. Expósito F, Lobos C, Roessler P. Educación, formación y trabajo: barreras para la inclusión en migrantes. In: Rojas Pedemonte N, Vicuña JT, editors. Migr. En Chile Evid. Mitos Una Nueva Real., Santiago de Chile: LOM ediciones; 2019, p. 107-42.

12. Bravo J. Mitos y realidades sobre el empleo en Chile. In: Rojas Pedemonte N, Vicuña JT, editors. Migr. En Chile Evid. Mitos Una Nueva Real., Santiago de Chile: LOM ediciones; 2019, p. 49-72.

13. ICIM, Colegio Médico de Chile, Servicio Jesuita a Migrantes, MICROB-R. Encuesta Sobre Covid-19 a Poblaciones Migrantes Internacionales En Chile. Santiago de Chile: 2020.

14. Aglipay M, Wylie JL, Jolly AM. Health research among hard-to-reach people: Six degrees of sampling. CMAJ .2015;187

(15):1145-9. https://dx.doi.org/10.1503/cmaj.141076

15. Faugier J, Sargeant M. Sampling hard to reach

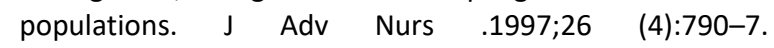
https://dx.doi.org/10.1046/j.1365-2648.1997.00371.x

16. Mercer A, Lau A, Kennedy C. For Weighting Online Opt-In Samples, What Matters Most? 2018.

17. Powers DA, Xie Y. Statistical Methods for Categorical Data Analysis. London, England: Academic Press, Inc; 2000.

18. Agresti A, Finlay B. Statistical Methods for Social Sciences. New Yersey, USA: Pearson Prentice Hall; 2009.

19. Stefoni C, Bonhomme M. Una vida en Chile y seguir siendo extranjeros. Si Somos Am Rev Estud Transfront .2014;14 (2):81-101. https://dx.doi.org/10.4067/S071909482014000200004

20. Servicio Jesuita a Migrantes. Criminalidad, Seguridad y Migración: Un análisis en el Chile actual. Santiago de Chile: 2020.

21. Jirón $P$, Ulriksen $C$, Margarit $D$, Imilan W. Trabajadores Móviles Digitales en Chile. Policy Brief - Ser Condiciones Empl En Chile En Contexto Crisis Sanit .2021

22. Fadnes LT, Møen KA, Diaz E. Primary healthcare usage and morbidity among immigrant children compared with non-immigrant children: A population-based study in $\begin{array}{llll}\text { Norway. BMJ Open } & \text {.2016;10 (6):1-8. }\end{array}$ https://dx.doi.org/10.1136/bmjopen-2016-012101

23. Hasanali S. Immigrant-Native Disparities in Perceived and Actual Met/Unmet Need for Medical Care. J Immigr Minor $\begin{array}{lll}\text { Health } & .2015 ; 17 & \text { (5):1337-46. }\end{array}$ https://dx.doi.org/10.1007/s10903-014-0092-x

24. Troncoso M, Troncoso CG, Link F. Situación Habitacional de las personas migrantes en algunas comunas urbanas de la Región Metropolitana y Antofagasta. Santiago de Chile: 2018 